\title{
Recovering smooth dynamics from time series with the aid of recurrence plots
}

\author{
Fatihcan M. Atay* and Yiğit Altıntaş \\ Mathematics Department, Koç University, Istinye 80860, Istanbul, Turkey \\ (Received 12 August 1998; revised manuscript received 8 January 1999)
}

\begin{abstract}
A graphical method based on recurrence plots is used in the reconstruction of the phase space from a time series of measurements. It is demonstrated that if the embedding delay and dimension are correctly chosen, the recurrence plot of a smooth dynamical system has a particularly simple form. It is shown how to use recurrence plots to determine the correct embedding parameters so that reliable quantitative information can be drawn about the system generating the time series. The average line length in the plot is shown to be directly related to the prediction horizon. Furthermore, it is a numerical characteristic of the embedded series independent of the threshold used in the plot. [S1063-651X(99)04206-3]

PACS number(s): 05.45.-a, 05.40.-a
\end{abstract}

\section{INTRODUCTION}

The method of delay coordinates is a well-established and widely-used tool to reconstruct the phase-space geometry of a chaotic system using only the observations of a single component. It forms the basis of many further analyses performed on the data, ranging from graphical representations to the calculation of numerical characteristics such as the Lyapunov exponents, correlation integrals, and fractal dimensions [1]. Since there is not a unique way to go about the reconstruction, guaranteeing the accuracy of the calculations is not a simple matter, however. Here we use a simple graphical tool to test how well the reconstructed geometry represents the dynamical characteristics of the system.

The delay-coordinate reconstruction is based on the idea that, given a scalar sequence $x_{1}, x_{2}, \ldots$, of measurements taken from a dynamical system at equally spaced time instants, the locus of the $d$-dimensional vectors

$$
\mathbf{v}_{k}=\left(x_{k}, x_{k-\tau}, \ldots, x_{k-(d-1) \tau}\right)
$$

will give a picture which is equivalent to the actual geometry of the attractor. Indeed, the embedding theorem of Takens ensures that the procedure will succeed for almost any choice of the embedding delay $\tau$ and a sufficiently large embedding dimension $d[2,3]$. (Generically, the necessary embedding dimension is not more than $2 n+1$, where $n$ is the dimension of the attractor.) For a finite data size, however, when it comes to estimating quantitative information from the reconstruction, such as the Lyapunov exponents, different choices of the embedding parameters can lead to different estimates [4]. The reason is that while the reconstruction is topologically equivalent to the actual attractor, there is not necessarily a diffeomorphic or isometric equivalence. In other words, the delay coordinates give a continuously deformed picture of the attractor, possibly distorting the tangent vectors and the distances in the structure. Several methods have appeared in the literature which address the problem of how to choose

\footnotetext{
*Author to whom correspondence should be addressed. Present address: Arçelik A. Ş.-Artesis, Tuzla 81719, Istanbul, Turkey. FAX: (216) 4233045 . Electronic address: atay@ arcelik.com.tr
}

the parameters for a "good" reconstruction of the phase space [1]. Our treatment of the problem emphasizes the faithful reconstruction of the differential structure and makes use of the recurrence plots introduced in Ref. [5].

The recurrence plot is a device displaying how the reconstructed trajectory comes close to itself. It has been used to test for stationarity [5] or chaos in time series [6], as well as to extract periodic orbits [7]. The plot is constructed as follows: Let $\delta$ be a fixed positive number, and define the array

$$
a_{i j}=\left\{\begin{array}{ccc}
1 & \text { if } & \left\|\mathbf{v}_{i}-\mathbf{v}_{i+j}\right\|<\delta \\
0 & \text { otherwise }
\end{array}, i, j=1,2, \ldots,\right.
$$

where the $\mathbf{v}_{k}$ are the vectors obtained after the embedding (1), and $\|\cdots\|$ denotes the usual Euclidean norm. The quantity $\delta$ is a measure of closeness and is usually expressed as a percentage of the diameter of the attractor or the standard deviation of the time series data. The recurrence plot is obtained by plotting a point on the $i$-j plane whenever $a_{i j}=1$. Notice that our definition (2) is slightly different and somewhat simpler than the one given in Ref. [5], but the underlying idea is the same. The resulting two-dimensional graph can contain intriguing patterns; however, we argue that much of these is an artifact of the way the embedding is done, and if the embedding parameters are correctly chosen, all one should see are simple horizontal line segments. Hence, the question of whether the reconstruction actually represents the true dynamics can be directly answered by looking at the recurrence plot.

We use ideas from the theory of smooth dynamical systems to deduce the type of patterns the recurrence plot can and cannot contain, and we distinguish good and bad embeddings by their corresponding plots. We test our conclusions by showing that the embeddings deemed bad by the recurrence plot test perform poorly in the calculation of the Lyapunov exponents and prediction of the time series. We also show that the average line length in the plot is largely independent of the choice of the threshold $\delta$, and so can be used as a numerical value summarizing the visual information offered by the plot. Finally, we investigate the relationship between the average line length, the largest Lyapunov exponent, and the prediction of the series. 


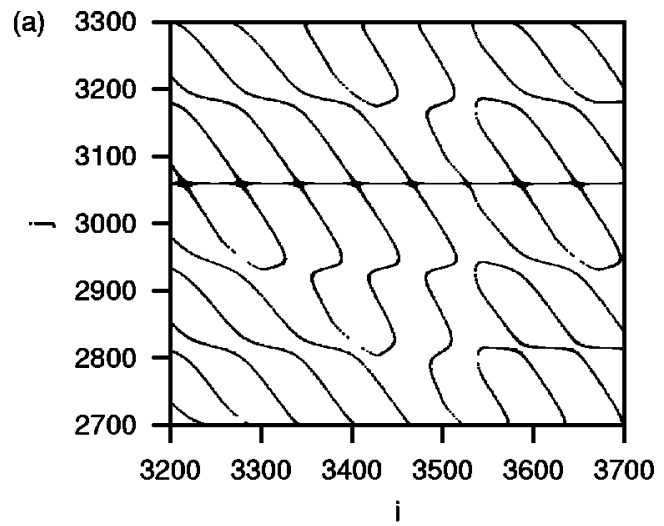

(c)

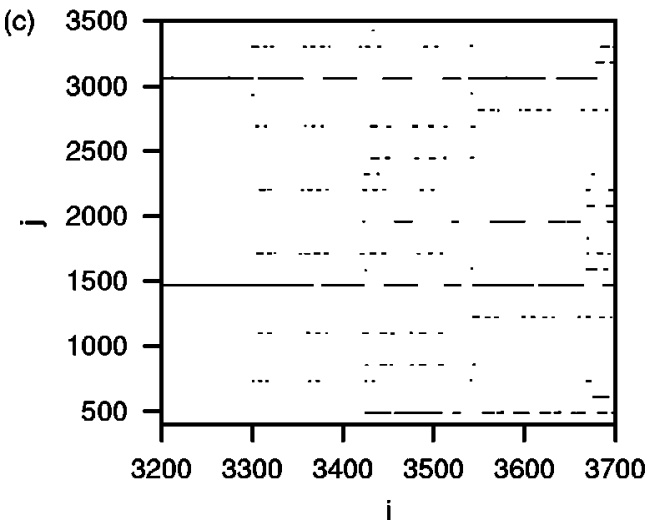

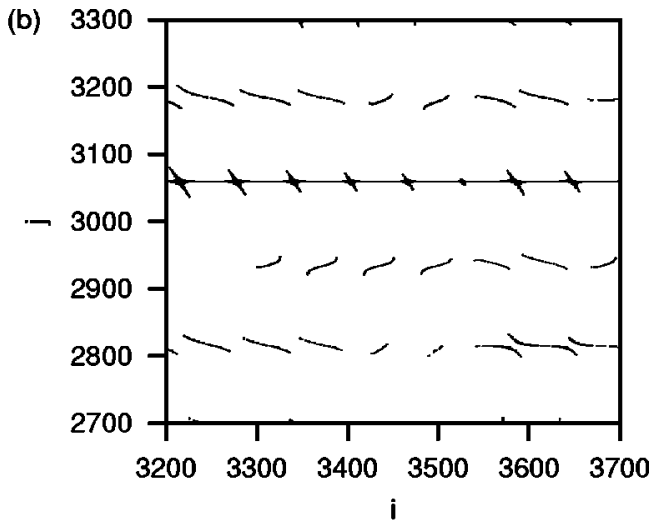

(d)

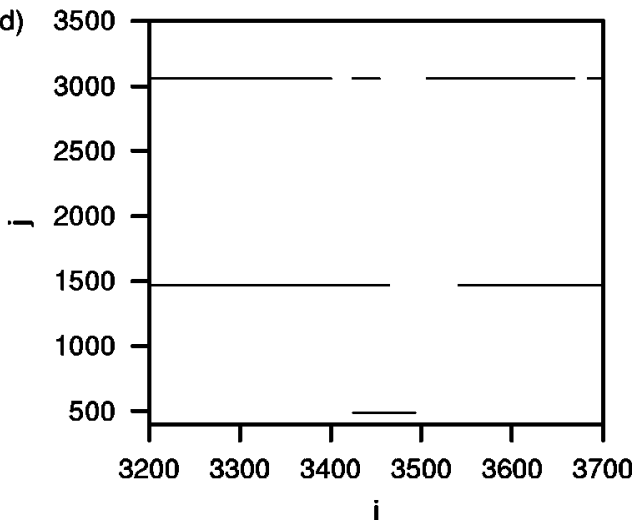

FIG. 1. Portion of the recurrence plot for the Rössler data. The parameters are (a) $d=1, \tau=1$, (b) $d=2, \tau=1$, (c) $d=6, \tau=1$, and (d) $d=6, \tau=8$. The value of $\delta$ is the same in all three figures.

\section{RECURRENCE PLOTS OF SMOOTH DYNAMICAL SYSTEMS}

We consider time series generated by smooth dynamical systems, and we assume that the time interval between measurements is sufficiently small to capture the smoothness of the trajectories. The important property we want to exploit for such systems is continuous (though possibly sensitive) dependence on initial conditions, of both the trajectories and also the derivatives. We also assume that the quantity $\delta$ in Eq. (2) is chosen sufficiently small so that the recurrence plot displays only local information, although the exact value of $\delta$ will be of little consequence for our purposes (see also Sec. IV).

By continuous dependence on initial conditions, if two trajectories come close in the phase space they will remain close for some time interval, and this will be reflected as a horizontal line segment in the recurrence plot. On the other hand, a nonhorizontal curve in the plot indicates two trajectories are close but their tangent vectors point in different directions, so that, say, the point $\mathbf{v}_{10}$ is close to $\mathbf{v}_{100}$, but $\mathbf{v}_{11}$ is close to $\mathbf{v}_{99}$ instead of $\mathbf{v}_{101}$. Such a situation is in violation of the continuity of the tangent vectors, and indicates that the phase-space reconstruction does not faithfully reproduce the differential structure. This typically occurs when the embedding dimension is too small to prevent the intersection of the trajectories, but can also occur if an incorrect choice of the embedding delay artificially squeezes the trajectories together. Although this first aspect has been noted before (e.g., Ref. [7]), the role played by the delay seems largely over- looked. We illustrate both situations with the aid of Figs. 1 and 2.

Figure 1 shows the recurrence plot of the data generated by the $x$-coordinate of the familiar Rössler system

$$
\begin{gathered}
\dot{x}=-y-z, \\
\dot{y}=x+a y, \\
\dot{z}=b+z(x-c),
\end{gathered}
$$

with $a=b=0.1$ and $c=14$, for an embedding delay $\tau=1$ and for different embedding dimensions. The nonhorizontal patterns disappear as the embedding dimension $d$ is increased from 1 to 6 [Fig. 1(a) through 1(c)]. This is as expected since the Rössler attractor has a (fractal) dimension between 2 and 3 , and choosing a large enough dimension would unfold the attractor. However, increasing the dimension is not always the solution. Consider the periodic signal shown in Fig. 2(a), which was studied in Ref. [8]. The recurrence plot of a periodic orbit should consist only of equally spaced horizontal lines, indicating periodic recurrence for all points. For this signal, however, a typical plot looks similar to Fig. 2(b), obtained for the values $d=3$ and $\tau=1$. Although the exact pattern depends also on the value of $\delta$, changing $\delta$ does not remove the nonhorizontal structures in the plot. By increasing the embedding dimension up to 10 , the authors in Ref. [8] succeed in removing some, but not all, of the nonhorizontal patterns. On the other hand, by the embedding theo- 

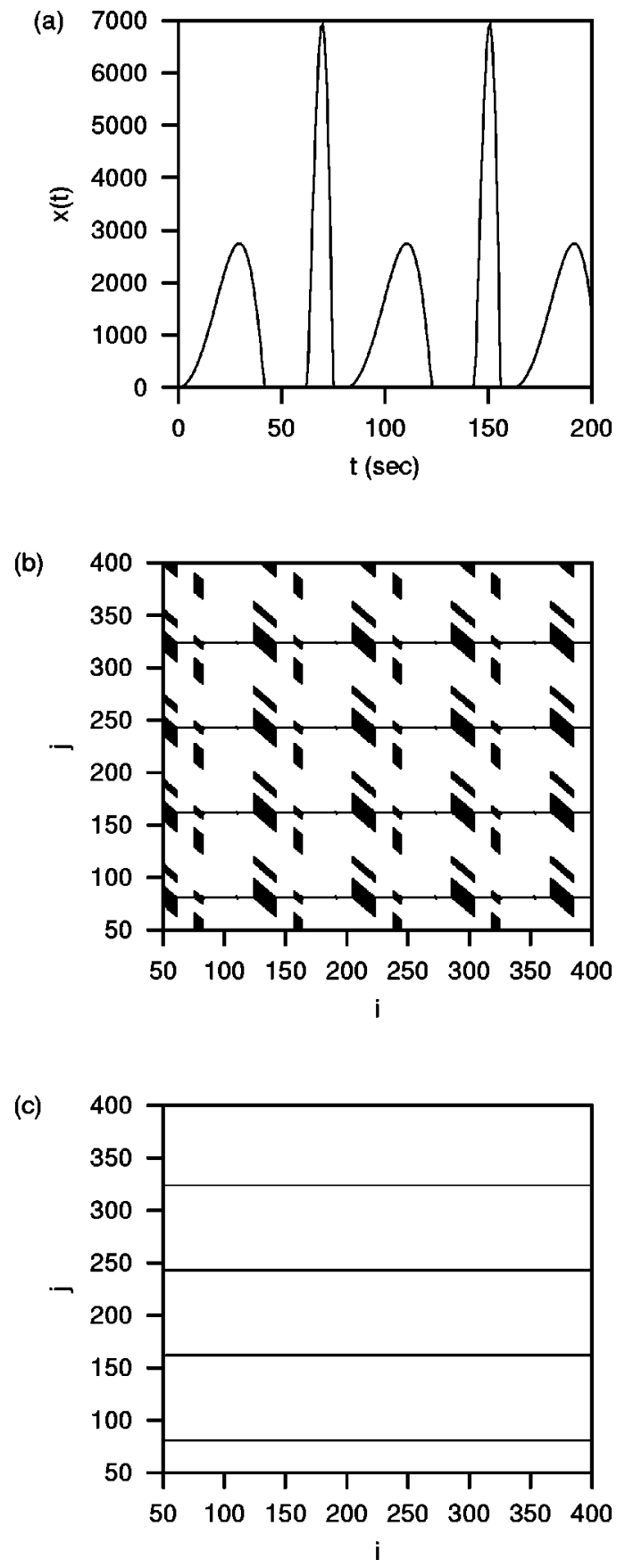

FIG. 2. Recurrence plots of the periodic function shown in (a) for the values (b) $\tau=1$, and (c) $\tau=10$. The embedding dimension is 3 for both plots.

rem, three dimensions should be sufficient to embed any periodic orbit (a one-dimensional closed curve). Clearly then, the problem lies not with the choice of the dimension but with the delay $\tau$. Figure 2(c) shows the recurrence plot when $\tau$ is increased to 10 , with $d=3$. The recurrence plot can thus help determine the correct choices for both the embedding dimension and the embedding delay.

Cleaning a recurrence plot from nonhorizontal patterns is a first step in the determination of the correct embedding parameters, but usually is not sufficient. A close inspection of Fig. 1(c) displays two other undesirable features: (1) isolated points, or very short lines and (2) short gaps frequently interrupting line segments. Shortness here is to be under- stood in terms of the time scale used in the measurements, and not as a simple count of points. Now such patterns in the plot is an indication of points in phase space coming close and then immediately moving away, or starting to move away but returning instantly, which imply widely varying derivatives at nearby points. However, inside a bounded attractor of a smooth system the derivatives are also bounded, and by choosing a sufficiently small $\delta$ it is possible to rule out the possibility of such behavior as seen in Fig. 1(c). In practice these may still show up because of the finite time interval between observations and the necessity to work with a reasonably large $\delta$ to reveal any patterns in a finite time series; nevertheless, they should be rare. For instance, the plot in Fig. 1(d) agrees better with our expectations of a smooth dynamical than Fig. 1(c) does. The only difference between the two plots is in the choice of the embedding delay. We would expect then that a "clean" recurrence plot such as Fig. 1(d) represents a better reconstruction of the attractor, and we test the assertion by calculating the Lyapunov exponents and the prediction errors.

\section{TESTING THE ASSERTIONS}

\section{A. Lyapunov exponents}

The notion of a recurrence plot is intimately related to the calculation of the average local Lyapunov exponents from time series: The basic idea in the calculation of the maximum exponent, for instance, is to find a pair of spatially nearby points in the attractor and follow their time evolution to measure the rate of divergence [4], until the points can no longer be considered close. Hence, short horizontal lines in the recurrence plot correspond to large local exponents. Clearly, both procedures are dependent on how the embedding is done (i.e., the choice of the dimension and the delay), as well as what the measure of closeness is [i.e., the quantity $\delta$ in Eq. (2)]. This gives a way to check our assertions as follows. We generate a time series from a system whose equations of motion are known, and construct a recurrence plot. The three parameters used in the plot are then fed to an algorithm based on Ref. [4] to calculate the largest Lyapunov exponent from the time series, and the result is compared to the exponent calculated directly from the equations of motion. The agreement between the two calculated exponents is an indication that the corresponding embedding parameters are correctly chosen. To ensure that the errors are not due to the shortness of the time series, data consisting of 20000 or more points are used in the calculations.

The comparison of the exponent is done for several qualitatively different systems. In all cases it is found that when the recurrence plot is "clean," in the sense of containing only (uninterrupted) horizontal line segments, the corresponding embedding parameters allow very accurate calculation of the exponents. For instance, using the parameters of Fig. 1(c) the calculated exponent is about 6 times as large as the true exponent, leading to a huge relative error, while those of Fig. 1(d) result in a relative error of less than $1 \%$. The fact that Fig. 1(c) results in an overestimation of the exponent gives further support to our discussion about the dynamics reflected in the plot-the single points and shorter segments in the figure falsely imply a high rate of local sepa- 

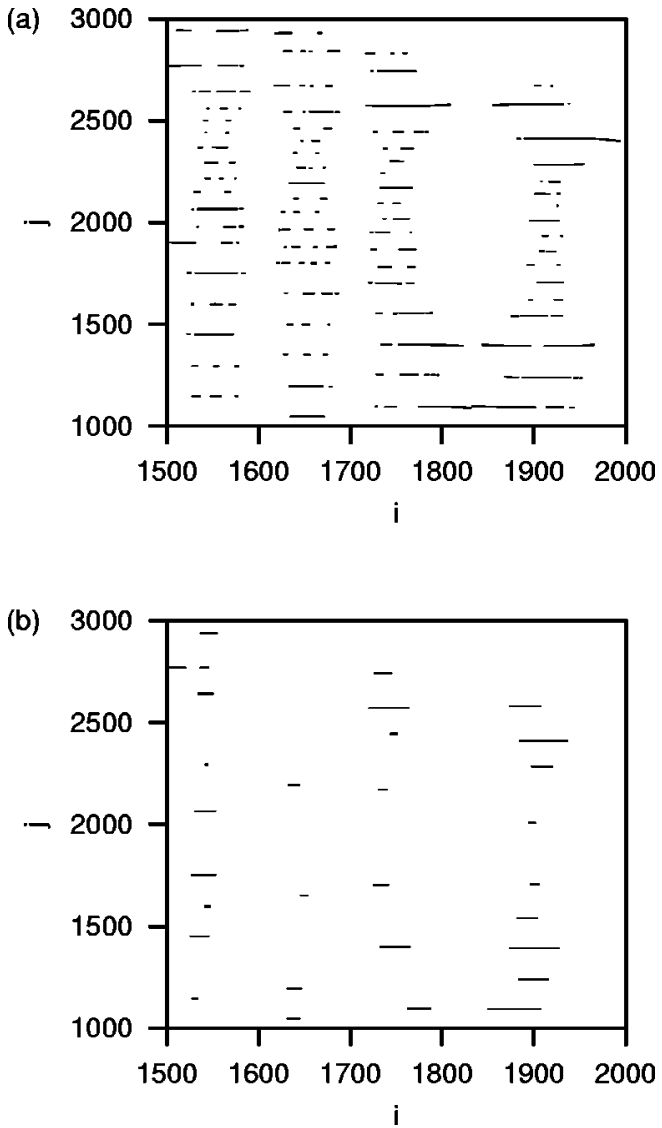

FIG. 3. Recurrence plots for the Hamiltonian system given by Eq. (4) for (a) $\tau=1$ and (b) $\tau=6$. The embedding dimension is 8 for both plots.

ration. Similar results were obtained with the Lorenz system (data not shown).

We also tested conservative systems, such as the one modelling two nonlinearly coupled anharmonic oscillators with the Hamiltonian

$$
\begin{aligned}
H= & \frac{1}{2}\left(p_{x}^{2}+p_{y}^{2}\right)+\frac{1}{2} x^{2}-0.05 x^{3}+0.00140625 x^{4}+0.72 y^{2} \\
& -0.0864 y^{3}+0.002916 y^{4}+0.1 x^{2} y^{2}
\end{aligned}
$$

where $x, y$ are the position coordinates and $p_{x}, p_{y}$ are the momenta. The exponent calculation for this system for different energy levels was discussed in Ref. [9]. Typical recurrence plots at a particular energy level are shown in Fig. 3. The parameters used in Fig. 3(a) result in a relative error of $160 \%$ in the exponent calculation, while the cleaner plot in Fig. 3(b) gives 4\% error. For all the energy levels considered in Ref. [9], it has been possible to estimate the exponent with $1-4 \%$ error using the parameters obtained from a clean recurrence plot.

\section{B. Prediction errors}

The phase-space neighbors can be used to predict the future evolution of a given point in the embedded time series [10]. For a chaotic system the prediction is necessarily a short-term one, and the prediction horizon is mainly determined by the Lyapunov exponents, although it also depends
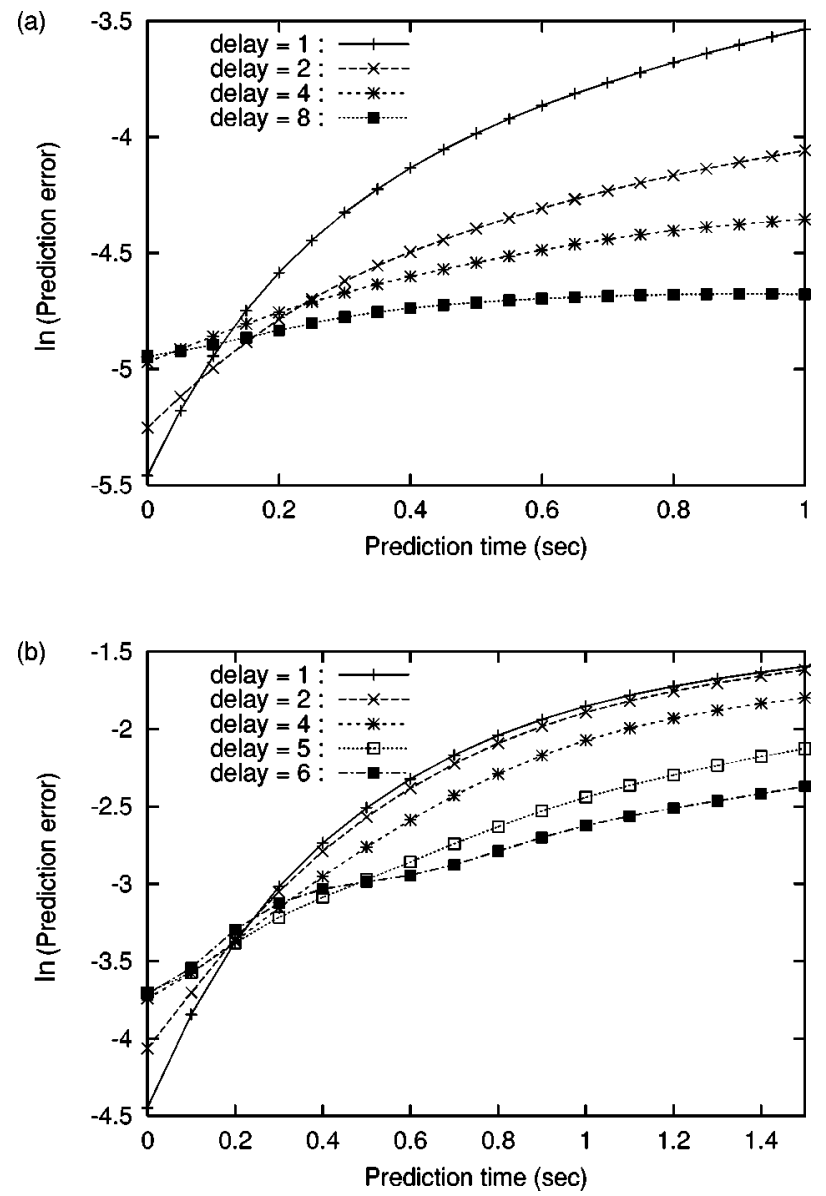

FIG. 4. Prediction errors for the Rössler (a) and the Hamiltonian (b) systems for various choices of the embedding delay.

on the way the embedding is done. To make the connection with the recurrence plots, as a predictor for a given point we use points in its $\delta$ neighborhood which are temporally in the past. Iterates of this neighbor are compared to the iterates of the given point, and the difference is divided by the standard deviation of the data, giving the normalized prediction error. Prediction is done for each point in the second half of the time series. In case a neighborhood contains more than one point, their average is used; in the case when it contains none, no prediction is made. Plotting the average prediction error against the prediction time shows how the error grows at each iterate as a result of chaos.

Figure 4 shows plots of the logarithm of the prediction error for the Rössler system and the Hamiltonian system given by Eq. (4), for various choices of the embedding delay. It is seen that the small delays of 1 and 2 initially give a smaller prediction error, but the rate of increase of error is much faster. Since the slopes of the lines are directly related to the largest Lyapunov exponent [10], the implication is that the use of these delay values would result in a large exponent estimate, confirming our earlier observation using recurrence plots. As the delay is increased, the error lines converge near a delay of 8 for the Rössler and 6 for the Hamiltonian system.

The ability to predict further into the future is an indication of the quality of the embedding, since it reflects the deterministic structure in the data. This information can also be obtained visually from the recurrence plot. At the end of 


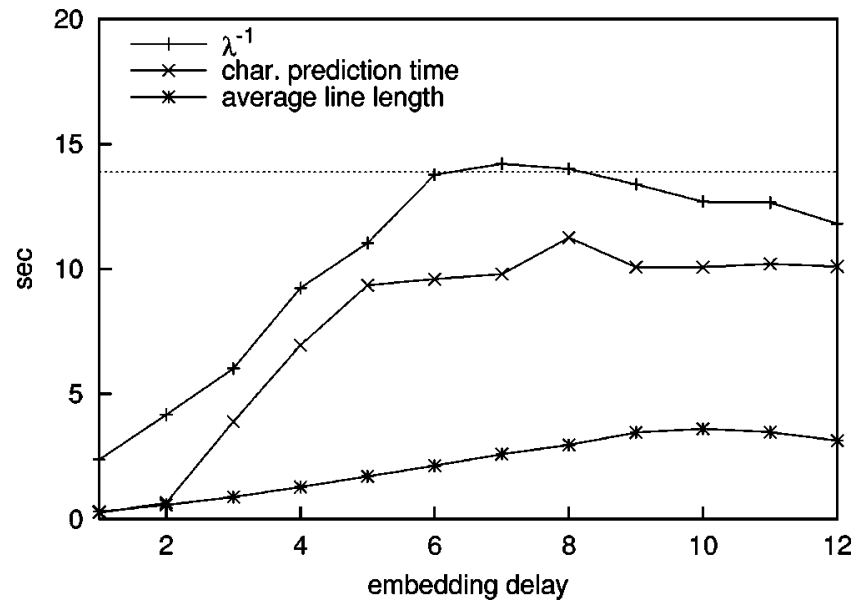

FIG. 5. Comparison of the inverse of the largest Lyapunov exponent $\lambda^{-1}$ (as calculated from the time series), characteristic prediction time $T_{e}$, and the average line length $L$ in the recurrence plot for the Rössler system. The dotted horizontal line shows the reciprocal of the true value of the largest exponent.

each horizontal line, a large prediction error is introduced. Consequently, long uninterrupted lines signal good prediction performance, whereas single points contribute largely to the prediction error. It is therefore not surprising that an embedding which results in a clean plot with long line segments will also lead to better forecasting. This also suggests to use the average line length as a numerical measure of the quality of embedding, which we discuss in the next section.

A word of caution may be in order: We have deliberately chosen examples of bad embeddings to illustrate that large errors are possible if the embedding is not done properly. In particular, our conclusion is not that long embedding delays are necessarily better than shorter ones, but if an embedding is unsatisfactory, because of either too small or too large a delay, then it shows in the recurrence plot.

By the results of the Lyapunov exponent and prediction error calculations, we conclude that a direct investigation of the recurrence plot can reliably tell whether the embedding parameters are correctly chosen. While it may not always be possible to obtain a completely clean plot due to the finite time interval between observations, the recurrence plot can still tell if an embedding is better than the others, within the limitations of the available data.

\section{LINE LENGTHS IN THE RECURRENCE PLOT}

To quantify the visual information offered by the recurrence plot, the average length $L$ of the horizontal lines can be useful. The foregoing discussion suggests that this quantity would be directly related to the prediction horizon and to the inverse of the largest Lyapunov exponent $\lambda^{-1}$. To facilitate comparison, we define a characteristic prediction time $T_{e}$ as the time it takes for the initial error to grow by a factor of $e$. Figure 5 summarizes the relation between $L, \lambda^{-1}$, and $T_{e}$ for the Rössler system for various choices of the embedding delay. The observation that the three curves have their maxima at slightly different locations can be attributed to the errors associated with each method in dealing with a finite time series. However, all three methods indicate that 8 or nearby delay values would give an acceptable embedding,

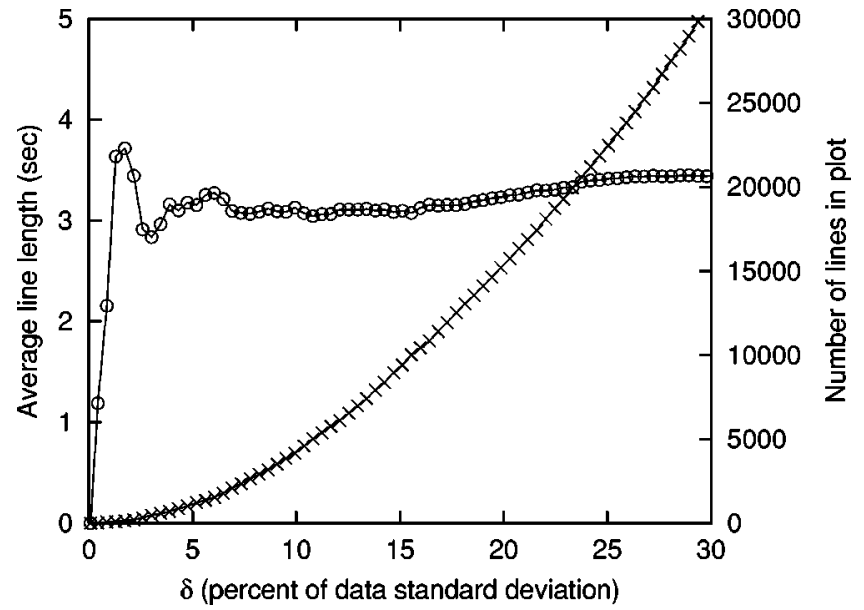

FIG. 6. As the threshold $\delta$ is increased, the number of lines $(X)$ in the recurrence plot greatly increases, but the average line length $(\bigcirc)$ is virtually constant. A 20000 -point time series from the Rössler system is used in the calculations.

while a value less than 3 or 4 would not.

It might appear that the threshold $\delta$ used in the recurrence plot would affect the line lengths, so one might object to the use of the average line length $L$ as an invariant of the dynamics. It turns out that this is not the case. The two effects of increasing $\delta$ so that more points appear on the plot are, on the one hand, making existing lines longer, while on the other hand creating new lines which are necessarily short by the continuity of the process. These two counterbalance each other to render the average line length constant. Figure 6 illustrates this for the Rössler system: As $\delta$ is increased the number of horizontal lines in the plot greatly increases, while the average line length is virtually unchanged. Therefore, the selection of the threshold $\delta$ is not critical for the conclusions derived from the recurrence plot. Of course, if $\delta$ is chosen too large, then the plot will contain too many points to discern any patterns; while if it is chosen too small, the plot will contain too few lines to be statistically significant. As suggested by Fig. 6, a value of $\delta$ in the range of $5-30 \%$ of the standard deviation of data seems safe for many practical data sizes.

With the existence of the long segments and the disappearance of the shorter ones, the average length of the line segments increases towards a good embedding. Being independent of the threshold $\delta$, the average line length can thus serve as a numerical measure in determining the quality of the embedding.

\section{CONCLUSION}

In quantitative studies of time series using reconstructed phase-space it is often crucial that the differential structure is accurately represented. For this purpose it is important that the parameters used in the reconstruction are properly chosen. Among these parameters, the determination of the embedding dimension usually does not present much difficulty. In fact, increasing the dimension beyond the sufficient quantity $2 n+1$ does not change the reconstructed space much. The choice of the embedding delay, on the other hand, while not changing the topology, can have a profound effect on the 
differential structure. The recurrence plots can be used to display whether the phase-space behavior of the embedded time series is consistent with the smoothness of the actual system. The effects of the incorrect choices of the embedding parameters can be seen visually as deviations from a simple structure consisting of horizontal lines. Moreover, the average line length can serve as a numerical guide for comparing different embeddings, or for quantifying chaos in general. The results turn out to be insensitive to the choice of the threshold $\delta$, which is the only extra parameter used in the plot. Once properly constructed, graphical tools such as the recurrence plot can be invaluable for gaining more insight into the dynamics of the system, especially since visualization becomes difficult beyond three dimensions.
[1] H. D. I. Abarbanel, R. Brown, J. J. Sidorowich, and L. S. Tsimring, Rev. Mod. Phys. 65, 1331 (1993).

[2] N. H. Packard, J. P. Crutchfield, J. D. Farmer, and R. S. Shaw, Phys. Rev. Lett. 45, 712 (1980).

[3] F. Takens, in Dynamical Systems and Turbulence, Vol. 898 of Lecture Notes in Mathematics, edited by D. A. Rand and L. S. Young (Springer-Verlag, New York, 1981), pp. 366-381.

[4] A. Wolf, J. B. Swift, H. L. Swinney, and J. A. Vastano, Physica D 16, 285 (1985).

[5] J. P. Eckmann, S. O. Kamphorst, and D. Ruelle, Europhys. Lett. 4, 973 (1987).
[6] C. G. Gilmore, J. Econ. Behav. Organ. 22, 209 (1993).

[7] G. B. Mindlin, H. G. Solari, M. A. Natiello, R. Gilmore, and X.-J. Hou, J. Nonlinear Sci. 1, 147 (1991).

[8] M. Koebbe and G. Mayer-Kress, in Nonlinear Modeling and Forecasting, edited by M. Casdagli and S. Eubank (AddisonWesley, Redwood City, CA, 1992), p. 361.

[9] F. M. Atay and E. Yurtsever, Chem. Phys. Lett. 276, 282 (1997).

[10] J. D. Farmer and J. J. Sidorowich, Phys. Rev. Lett. 59, 845 (1987). 